\section{Association between cystoid spaces on indocyanine green hyperfluorescence and optical coherence tomography after vitrectomy for diabetic macular oedema}

S Yoshitake, T Murakami, A Uji, K Ogino, T Horii, M Hata, S Arichika, K Nishijima and N Yoshimura

\begin{abstract}
Purpose To study retrospectively the characteristics of residual indocyanine green (ICG) fluorescence after ICG-assisted vitrectomy and the association with spectraldomain optical coherence tomography (SD-OCT) findings in diabetic macular oedema (DMO).

Methods Thirteen consecutive eyes of $\mathbf{1 2}$ patients for whom fundus near-infrared fluorescence and $20^{\circ}$ retinal sectional images were obtained using HRA2 and Spectralis $\mathrm{OCT}$, respectively, 5 days after vitrectomy combined with ICG-assisted inner limiting membrane peeling for DMO. The relationship between the characteristics of the ICG hyperfluorescence and the cystoid spaces in the outer plexiform layer (OPL) on SD-OCT images was evaluated.

Results A total of 390 well-demarcated areas of ICG hyperfluorescence were delineated on $20^{\circ}$ radial OCT scans dissecting the fovea 5 days after vitrectomy. The areas of ICG hyperfluorescence in the parafovea or perifovea were significantly smaller than those at the fovea. Most areas of hyperfluorescence were irregularly shaped in the parafovea and perifovea, whereas 18 of 38 areas of hyperfluorescence were round or oval at the fovea $(P<0.001)$. SD-OCT delineated the cystoid spaces in the OPL in 73 areas of hyperfluorescence that were round or oval and accompanied by dark spots more frequently than that without
\end{abstract}

cystoid spaces on OCT images $(P<0.001$ and $P=0.002)$. Of the 123 cystoid spaces in the OPL on OCT images, 44 did not have ICG hyperfluorescence, had lower OCT reflectivity, and contained fewer hyperreflective foci than those with ICG hyperfluorescence $(P<0.001$ and $P=0.020$ ).

Conclusion The results provided novel interpretations of the ICG hyperfluorescence and its association with OCT characteristics of the cystoid spaces in DMO.

Eye (2014) 28, 439-448; doi:10.1038/eye.2013.290; published online 24 January 2014

Keywords: diabetic macular oedema; indocyanine green; optical coherence tomography; cystoid space

\section{Introduction}

Diabetic macular oedema (DMO) is a major cause of severe visual loss in diabetic retinopathy. ${ }^{1}$ Diabetes induces breakdown of the blood-retinal barrier (BRB), and the accumulation of blood components in extravascular spaces leads to the morphologic pathogenesis, that is, macular thickening and functional disturbances. ${ }^{2,3}$ Several treatments including photocoagulation, vitrectomy, steroids, and anti-vascular endothelial growth factor (VEGF) agents have been used clinically and provide some benefit for the visual function. ${ }^{4-8}$ However, the pathogenesis of DMO
Department of Ophthalmology and Visual Sciences, Kyoto University Graduate School of Medicine, Sakyo, Kyoto, Japan

Correspondence: T Murakami, Department of Ophthalmology and Visual Sciences, Kyoto University Graduate School of Medicine, 54 ShogoinKawaracho, Sakyo, Kyoto 606-8507, Japan. Tel: + 8175751 3250; Fax: + 81757520933 . E-mail:mutomo@ kuhp.kyoto-u.ac.jp

Received: 7 March 2013 Accepted in revised form: 19 November 2013 Published online: 24 January 2014 
is ill-defined, and treatments that satisfy both patients and clinicians have yet to be developed.

Pathohistology elucidates the microscopic findings in the retinal vasculature and retinal parenchyma in macular oedema associated with retinal vascular diseases. Trypsin digestion preparation showed that, in addition to pericyte loss, the endothelial cells also disappeared, the so-called acellular capillaries in patients with diabetic retinopathy; ${ }^{9}$ experimentally, the intercellular junctions where the tight junction and adherens junction are highly integrated are disrupted in VEGF-treated or diabetic rodents, ${ }^{10,11}$ suggesting the diversity of the pathogenesis in BRB breakdown. ${ }^{12}$ In addition, histologic studies have reported that cystoid spaces in eyes with macular oedema are the primary structural changes in the retinal parenchyma. ${ }^{13-15}$ Cystoid spaces reside mainly in the inner nuclear layers (INLs) and outer plexiform layer (OPL), ${ }^{16-18}$ although it remains to be determined how the cystoid spaces develop in these layers in DMO. Electron microscopy has shown intracytoplasmic swelling of the Müller cells in macular oedema and the accumulated extracellular fluids in the retinal parenchyma, ${ }^{13-15}$ although the differences in the cystoid spaces based on the clinical findings have not been reported.

Biomicroscopy was originally used to clinically diagnose DMO. Another modality, fluorescein angiography (FA), delineates the vascular lesions with higher contrast and various patterns of hyperfluorescence in the retinal parenchyma, that is, focal or diffuse leakage and pooling with petalloid or honeycomb-like patterns. ${ }^{16,17,19}$ Importantly, FA leakage is not always synonymous with retinal oedema, because it does not necessarily indicate retinal thickening. It has also been reported that the patterns of fluorescein pooling are associated with the location of the cystoid spaces in DMO that was shown to be controversial based on systemic analyses. ${ }^{16,17}$ Spectral-domain optical coherence tomography (SD-OCT) is a noninvasive, noncontact modality that facilitates acquisition of in vivo, sectional, high-resolution images of the retinal pathology. Recent publications have reported a correlation between the cystoid spaces with microvascular lesions, including microaneurysms, and OCT reflectivity and fluorescein pooling that suggests several patterns of pathogenesis in the cystoid spaces in DMO. ${ }^{20,21}$ However, it remains to be determined how the cystoid spaces become so diverse in appearance.

Indocyanine green (ICG), which is excited by light with a longer wavelength, has been used to diagnose choroidal vascular diseases including age-related macular degeneration. As the basement membranes, such as the anterior lens capsule or the retinal inner limiting membrane (ILM), can also be stained and visualized by ICG, the dye has been used widely for successful ILM peeling in macular diseases including macular holes, epiretinal membranes, and macular oedema associated with retinal vascular diseases. ${ }^{22-27}$ In addition to the retinal toxicity of $\mathrm{ICG}^{28}$ several morphologic patterns of residual ICG several months after vitrectomy performed to treat macular holes have been reported: the area of the macular hole, residual ILM, stardust-like hyperfluorescence especially around the retinal arterioles, and arcuate hyperfluorescence along the nerve fibres from the ganglion cells. ${ }^{29-32}$ Only a few publications have reported the presence of ICG hyperfluorescence in macular oedema associated with retinal vascular diseases, ${ }^{33,34}$ although its characteristics are ill-defined and should be elucidated.

In this study, we showed for the first time the characteristics of well-demarcated ICG hyperfluorescence 5 days after ICG-assisted vitrectomy for DMO and compared them with the hyperfluorescence seen on FA images and the cystoid spaces on the SD-OCT images.

\section{Materials and methods}

\section{Patients}

We retrospectively reviewed 13 eyes of 12 patients (mean, $66.1 \pm 9.2$ years; range, $42-80$ ) who underwent vitrectomy combined with ICG-assisted ILM peeling for DMO at the Department of Ophthalmology, Kyoto University Hospital, between June 2011 and July 2012. Eyes were included that had centre-involved DMO and for which good-quality near-infrared autofluorescence and SDOCT images were obtained before and after surgery. ${ }^{35}$ The major exclusion criteria were the presence of any chorioretinal diseases, severe media opacity, a history of treatment for DMO or cataract surgery within 3 months of the start of the study, and a history of any other major surgery other than cataract extraction within 1 year of the start of the study. All research and measurements adhered to the tenets of the Declaration of Helsinki. The ethics committee of Kyoto University Graduate School of Medicine approved the study protocol.

\section{Surgical procedure}

A standard 23-gauge pars plana vitrectomy was performed in all eyes. After core vitrectomy, the residual posterior vitreous cortex was removed. While the infusion port was clipped, a small amount $(0.1-0.3 \mathrm{ml})$ of $0.25 \%$ ICG was sprayed onto the macular surface to visualize the ILM. Immediately after resuming the infusion line, the residual dye in the vitreous cavity was aspirated. The stained ILM was peeled using forceps in an area of 3 disc diameters or larger centred on the fovea. 
Ten phakic eyes underwent a standard cataract surgery (phacoemulsification, aspiration, and intraocular lens implantation). At the end of surgery, $20 \mathrm{mg}$ of triamcinolone acetonide was injected into the subTenon's capsule.

\section{ICG fluorescence}

After measuring the best-corrected visual acuity and performing fundus biomicroscopy, ICG fluorescence images of the macula were obtained using a scanning laser ophthalmoscope (Heidelberg Retina Autograph 2 (HRA2); Heidelberg Engineering, Heidelberg, Germany) preoperatively or postoperatively as described previously. ${ }^{31,33,34}$ Briefly, an macular area $30 \times 30^{\circ}$ was scanned using an 800-nm filter and excitation with the 787-nm diode laser preoperatively and a minimum of 5 days postoperatively. The images were averaged to improve the signal-to-noise ratio. Eleven eyes underwent FA preoperatively using the HRA2.

We evaluated several characteristics of welldemarcated ICG hyperfluorescence (area, position, shape, and presence of dark spots) on $20^{\circ}$ radial OCT scans in a clockwise manner 5 days after vitrectomy. First, the margin of the ICG hyperfluorescence was traced manually, and the area was quantified using image processing software (Photoshop, Adobe Systems, San Jose, CA, USA). The position of the centre of the ICG hyperfluorescence was classified into the fovea (central $1 \mathrm{~mm})$, parafovea (1-3 mm), and perifovea (3-6 mm) according to the Early Treatment Diabetic Retinopathy Study grid. The hyperfluorescence was described as round, oval, or irregular depending on the properties of the margin. We often found dot-like or spot-like hypofluorescence within well-demarcated ICG hyperfluorescence that we defined as dark spot(s) in this study.

\section{Optical coherence tomography}

Retinal sectional images of the macula were acquired and evaluated using SD-OCT (Spectralis OCT, Heidelberg Engineering) infrared images before and after vitrectomy. Radial scans $\left(20^{\circ}\right)$ centred on the presumed fovea were obtained in a clockwise manner. We counted the cystoid spaces in the OPL on all radial OCT scans and excluded any redundancy between individual scans. The OCT reflectivity levels of the cystoid spaces were calculated as reported previously. ${ }^{29}$ Briefly, after the margin of each cystoid space was determined manually, the averaged signal intensity was quantified using the Photoshop software. We also evaluated the presence of hyperreflective foci in the individual cystoid spaces. We thus investigated the relationship between the characteristics of well-demarcated ICG hyperfluorescence and the cystoid spaces in the OPL on the SD-OCT images.

\section{Statistical analysis}

The results are expressed as the mean \pm SD. Student's $t$-test was used to compare the quantitative data populations with normal distributions and equal variance. The data were analyzed using Mann-Whitney $U$-test for populations with nonnormal distributions or unequal variance. Significant differences in the sampling distributions were determined using the $\chi^{2}$-test or Fisher's exact test. $P<0.05$ was considered significant.

\section{Results}

\section{Characteristics of ICG hyperfluorescence}

When we investigated the characteristics of ICG hyperfluorescence after vitrectomy combined with ICGassisted ILM peeling, we found that the near-infrared autofluorescence was distributed diffusely, with the peak in the macula before surgery (Supplementary Figure 1A), whereas well-demarcated hyperfluorescence was delineated in the macula within 1 week after vitrectomy (Figure 1 and Supplementary Figure 1). This suggested that the hyperfluorescence corresponded to the residual ICG that was used to visualize the ILM, as reported previously. ${ }^{33}$ Some retinal capillaries were also accompanied by hyperfluorescence at the same time points (Figure 1). These signals gradually disappeared within a few months postoperatively (Supplementary Figure 1). Nerve fibres also presented intense signals of ICG fluorescence, as reported in eyes after ICG-assisted vitrectomy (Supplementary Figure 1). ${ }^{30,31}$ Stardust-like dot fluorescence, which emerged within a few months, accumulated along the retinal vasculature or optic disc (Supplementary Figure 1D). ${ }^{29,31}$

The ICG hyperfluorescence was diverse in size and shape and sometimes accompanied by dark spots. Some areas of hyperfluorescence were round or oval, and some were irregular and often accompanied by a higher intensity ICG signal (Figure 1). Intriguingly, 18 (47.4\%) areas of ICG hyperfluorescence within the central $1 \mathrm{~mm}$ were round or oval, whereas most ICG hyperfluorescence in the parafovea or perifovea $(91.4 \%$ and $98.3 \%$, respectively) were irregular (Table 1).

The hyperfluorescence around the fovea was often oval with the longer main axis radiating at the fovea. The areas of ICG hyperfluorescence were significantly larger at the fovea than in the parafovea or perifovea (Table 1). Based on these findings, we speculated that well-demarcated ICG hyperfluorescence corresponds to the cystoid spaces 




Figure 1 Resolution of well-demarcated indocyanine green (ICG) hyperfluorescence in the early phase after vitrectomy for diabetic macular oedema. (a) Well-demarcated ICG hyperfluorescence is side by side mainly in the temporal hemifield at 1 day after ICGassisted vitrectomy. The fluorescence has gradually faded in several places over time. At (b) 2 days, (c) 5 days, and (d) 2 weeks after surgery. (e) Round or oval ICG hyperfluorescence is seen at the fovea (white arrows) and sometimes contains dark spots (red arrow). (f) In contrast, the fluorescence in the perifovea is smaller and irregular (red arrow, large dark spot; red arrowhead, fine dark spot). (e, f) Magnified images of (c).

in the OPL rather than in the INL. The number of areas of hyperfluorescence in the nasal subfield was approximately one third of that in the temporal subfield (Supplementary Table 1). Single or multiple dark spots were present in 135 areas of ICG hyperfluorescence (Figure 1).

\section{Association of ICG hyperfluorescence with FA and SD-OCT}

The preoperative diffuse leakage of fluorescein sodium tended to be associated exclusively with ICG hyperfluorescence on postoperative day 5 in 11 eyes in which FA was performed preoperatively (Figure 2). In addition, some ICG hyperfluorescence 5 days postoperatively corresponded to petalloid patterns of foveal fluorescein pooling in preoperative FA, and most honeycomb-like fluorescein pooling was not colocalized to ICG hyperfluorescence (Figure 2). Interestingly, smaller areas of ICG fluorescence were often around the edge of the macular thickening (Figure 3).

We further investigated the relationship between ICG hyperfluorescence and cystoid spaces in the OPL on the 
Table 1 Characteristics of well-demarcated indocyanine green hyperfluorescence 5 days after ICG-assisted vitrectomy for diabetic macular oedema

\begin{tabular}{|c|c|c|c|c|}
\hline & Fovea & Parafovea & Perifovea & P-value \\
\hline No. of areas of ICG hyperfluorescence & 38 & 174 & 178 & \\
\hline Size of areas of ICG hyperfluorescence $\left(\times 10^{3} \mu \mathrm{m}^{2}\right)$ & $150.15 \pm 155.90$ & $62.30 \pm 53.30^{*}$ & $39.85 \pm 32.38^{* * * * * *}$ & \\
\hline \multicolumn{5}{|l|}{ Shapes of ICG hyperfluorescence } \\
\hline Round or oval & 18 & 15 & 3 & \\
\hline Irregular & 20 & 159 & 175 & $<0.001$ \\
\hline \multicolumn{5}{|l|}{ Cystoid spaces on SD-OCT } \\
\hline Present & 20 & 42 & 11 & \\
\hline Absent & 18 & 132 & 167 & $<0.001$ \\
\hline
\end{tabular}

Abbreviations: ICG, indocyanine green; SD-OCT, spectral-domain optical coherence tomography.

${ }^{*} P<0.01$ vs fovea.

${ }^{* *} P<0.001$ vs fovea.

$* * * P<0.001$ vs parafovea.
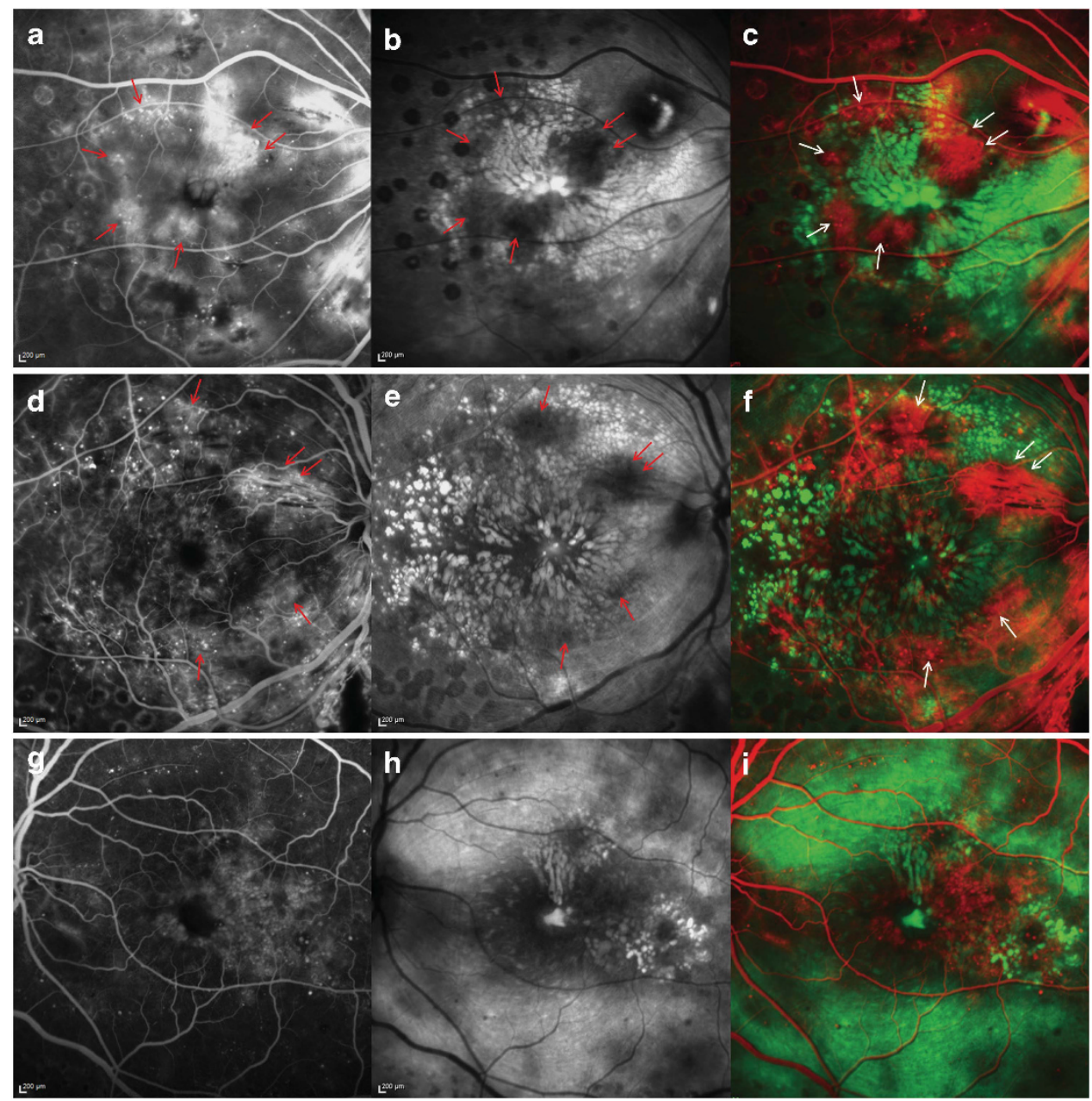

Figure 2 Well-demarcated indocyanine green (ICG) hyperfluorescence is associated exclusively with diffuse leakage in the preoperative fluorescein angiography (FA) image in diabetic macular oedema (DMO). Two representative cases that underwent ICGassisted vitrectomy for DMO. Diffuse leakage (arrows in a, d) in the FA image is seen in the areas with ICG hypofluorescence obtained 5 days after ICG-assisted vitrectomy (arrows in b, e). (c, f) In the superimposed images of the two cases, red indicates the FA image and green indicates the ICG fluorescence signal. $(\mathrm{g})$ The third case has fluorescein pooling with a honeycomb-pattern temporally that did not correspond to (h), ICG hyperfluorescence. (i) Superimposed image. 


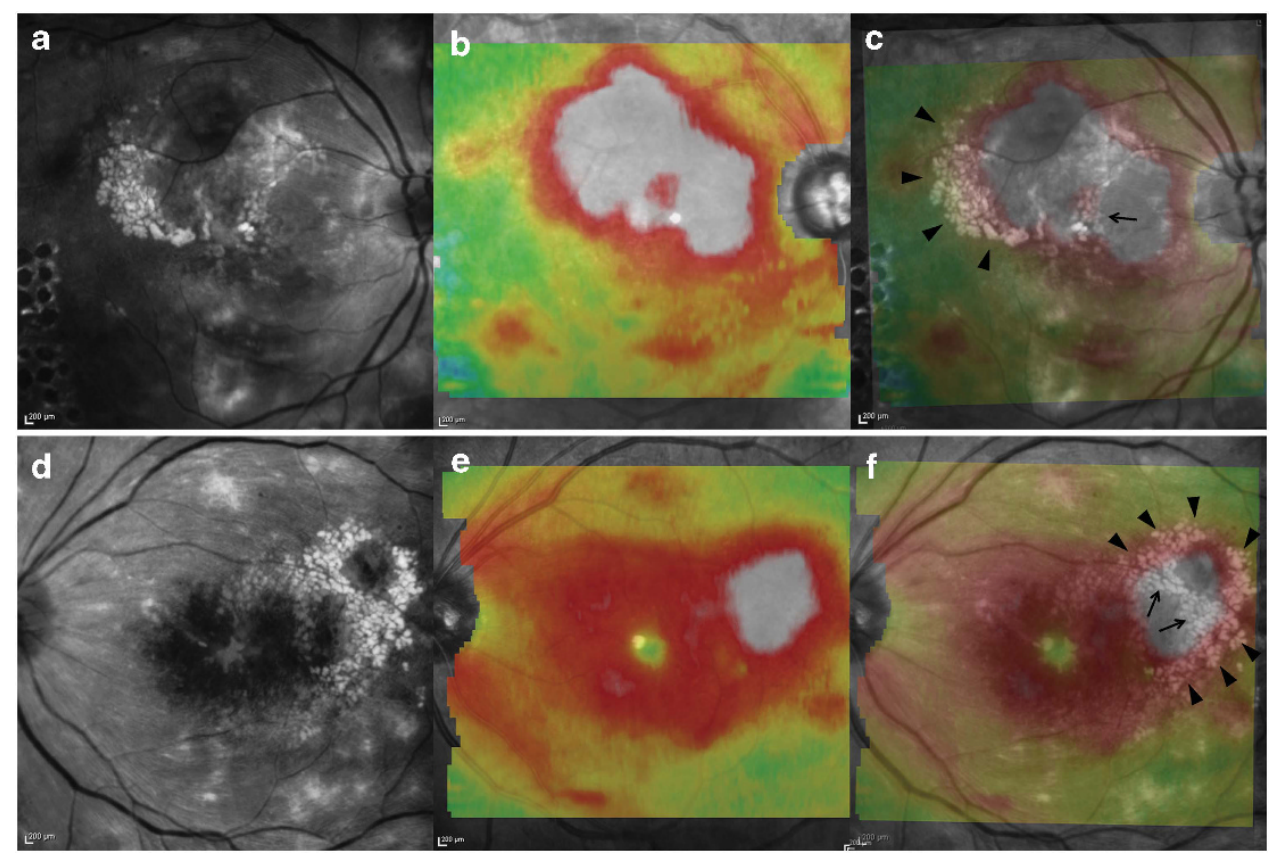

Figure 3 Indocyanine green (ICG) hyperfluorescence around the margin of macular thickening in diabetic macular oedema (DMO). (c) Some smaller areas of ICG hyperfluorescence with higher intensity are around the margin of macular thickening (arrowheads), whereas others are within the thickened areas (arrow) in two cases that underwent ICG-assisted vitrectomy for DMO. (a, d) ICG fluorescence 5 days after surgery. (b, e) OCT map images on the same day. (c, f) Superimposed images.

SD-OCT images (Figure 4 and Supplementary Figure 2). Spectralis OCT showed cystoid spaces in the OPL in 73 $(18.7 \%)$ corresponding areas of ICG hyperfluorescence.

The cystoid spaces in the OPL on SD-OCT images were seen in $20(52.6 \%)$ areas of ICG hyperfluorescence at the fovea, whereas only $11(6.2 \%)$ areas of hyperfluorescence in the perifovea had cystoid spaces in the OPL, with significant differences in the distribution $(P<0.001$; Table 1 and Supplementary Table 1). Cystoid spaces in the OPL were observed by Spectralis OCT in round or oval areas of ICG hyperfluorescence more frequently than in irregular areas $(47.2 \%$ vs $15.8 \%, P<0.001$; Supplementary Table 2). ICG hyperfluorescence with dark spots was also accompanied by cystoid spaces in the OPL more frequently than those without dark spots (27.4\% vs $14.1 \%, P=0.002$; Supplementary Table 2).

Spectralis OCT showed 123 cystoid spaces in the OPL on $20^{\circ}$ radial scans in a clockwise manner, and $79(64.2 \%)$ cystoid spaces had well-demarcated ICG hyperfluorescence (Figure 4, Supplementary Table 3, and Supplementary Figure 2). The OCT reflectivity levels were significantly higher in the cystoid spaces with ICG hyperfluorescence than in those without hyperfluorescence $(P<0.001$; Supplementary Table 3$)$. Hyperreflective foci were also seen in the cystoid spaces with hyperfluorescence more frequently than those without it $(P=0.020$;

Supplementary Table 3).

\section{Discussion}

In this study, we showed for the first time the characteristics of ICG hyperfluorescence and the association with the cystoid spaces on SD-OCT images in eyes that underwent ICG-assisted vitrectomy for DMO. As ICG has both hydrophilic and lipophilic properties, ${ }^{36}$ ICG is believed to bind to lipoproteins and to a lesser extent serum proteins including albumin, and ICG is deposited in the extracellular matrix containing the basement membrane. ${ }^{36-39}$ Well-demarcated ICG hyperfluorescence during the early postoperative phase might represent lipoproteins in the cystoid spaces, ${ }^{33}$ whereas it resolved within a few months. In contrast, stardust-like dot hyperfluorescence emerged a few months after vitrectomy, especially at the margin of the retinal vasculature and the optic disc, that seemed to correspond to the ICG phagocytosed by microglia or macrophages.

Several patterns of ICG hyperfluorescence were observed 5 days after ICG-assisted vitrectomy: well-demarcated fluorescence mainly in the macula, diffuse and faint fluorescence in any area, staining of retinal capillaries in the limited areas, and fluorescence along the nerve fibres from the ganglion cells. When we focussed on the first pattern of ICG hyperfluorescence, we considered several explanations: lipoproteins in the 




Figure 4 Association between indocyanine green (ICG) hyperfluorescence and optical coherence tomographic characteristics of cystoid spaces in diabetic macular oedema (DMO) treated with ICG-assisted vitrectomy. (a) An ICG fluorescence image obtained 5 days after ICG-assisted vitrectomy shows well-demarcated ICG hyperfluorescence at the fovea and in the temporal areas; (e) hyperfluorescence resolved at 2 weeks after surgery. In this case, most cystoid spaces on the optical coherence tomography (OCT) images got smaller but persisted. The red squares in (b, c, g) correspond to the red lines in (a) and (e) that indicate cystoid spaces in the outer plexiform layer (OPL) accompanied by ICG hyperfluorescence. The cystoid spaces with higher OCT reflectivity and hyperreflective foci at the fovea had persistent hyperfluorescence at 2 weeks (e, g); however, the fluorescence resolved in the cystoid spaces with lower OCT reflectivity (e, f). ICG hyperfluorescence in the areas outlined in yellow is not accompanied by definite cystoid spaces but with thickening in the OPL and outer nuclear layer in the OCT images (a, d, e, g, h). The cystoid spaces with lower OCT reflectivity in the areas outlined in blue did not have ICG hyperfluorescence (a, d, e, h).

cystoid spaces; deposits of serum proteins or lipids that have a higher affinity for ICG; and ICG intake by retinal cells including the Müller cells. The areas of this welldemarcated hyperfluorescence at the fovea were almost the same as those of fluorescein pooling of cystoid spaces in DMO, and the shapes in $18(47.4 \%)$ of 38 areas of ICG hyperfluorescence were round or oval at the fovea, which was similar to fluorescein pooling. Considering these findings, it is a reasonable explanation that ICG hyperfluorescence at the fovea represents lipoproteins in the cystoid spaces. In addition, the areas of fluorescence gradually decreased towards the periphery that is similar to the patterns of the cystoid spaces in the OPL rather than those in the INL seen on SD-OCT. The cystoid spaces in the OPL around the fovea radiated from the fovea and were oval with the longer major axis seen on the FA and OCT images, whereas most cystoid spaces in the INL were smaller on SD-OCT, with honeycomb-like fluorescein pooling on the FA images. ${ }^{16}$ These findings encouraged us to speculate that well-demarcated ICG hyperfluorescence corresponds to cystoid spaces in the
OPL rather than those in the INL pathohistologically. This might be further supported by the findings that ICG hyperfluorescence did not have the double layer and that the honeycomb-pattern fluorescein pooling was not colocalized to the ICG hyperfluorescence (Figure 2).

We also compared ICG hyperfluorescence and cystoid spaces in the OPL on SD-OCT and found that 73 areas of fluorescence had a cystoid space in the OPL of the corresponding area, whereas 317 areas of fluorescence did not. OCT showed cystoid spaces, depending on the optical differences between the content of the cystoid spaces and the retinal parenchyma. We thus hypothesized that 317 areas of ICG hyperfluorescence represent lipoproteins in the cystoid spaces that were not seen on the SD-OCT images. OCT often showed that relatively thicker OPLs and ONLs with heterogeneity of the reflectivity were accompanied by an undulated Henle's layer in the areas with well-demarcated ICG hyperfluorescence. This suggested that the cystoid spaces with the same OCT reflectivity as the surrounding retinal parenchyma were delineated on ICG fluorescence images 
but not on SD-OCT. This might agree with the finding that the cystoid spaces seen on SD-OCT images with ICG hyperfluorescence had higher levels of OCT reflectivity than those without fluorescence.

Of the 123 cystoid spaces on SD-OCT, 44 were not accompanied by well-demarcated ICG hyperfluorescence and had lower OCT reflectivity in the cystoid spaces. In this study, diffuse leakage seen on the FA images was associated exclusively with the ICG hyperfluorescence 5 days after ICG-assisted vitrectomy. We thus speculated that some of these cystoid spaces were absorbed after intervention (Supplementary Figure 2), whereas others persisted with higher rates of washing out of ICGbinding lipoproteins from the cystoid spaces (Figure 4). We considered a few possible mechanisms regarding the resolution. We first hypothesized that the diffuse leakage on FA indicates the origin of the extravasated blood components, whereas ICG hyperfluorescence represents the absorption of smaller molecules from less damaged capillaries and a concomitant increase in the lipoprotein concentration. This might agree with the finding that the ICG hyperfluorescence was around the border of the macular thickening in this study and in a previous publication that documented ICG fluorescence at the border between the occluded and unaffected areas in branch retinal vein occlusion. ${ }^{34}$ A second possible explanation was that the hyperfluorescence in the FA images that corresponded to the extravasation of smaller molecules promotes dilution of the lipoproteins in the cystoid spaces. A recent study reported a negative association between fluorescein pooling and OCT reflectivity in the foveal cystoid spaces in $\mathrm{DMO}^{21}$ that encouraged us to speculate that higher levels of fluorescein pooling mean the extravasation of smaller molecules rather than macromolecules and a concomitant decrease in the concentration of lipoproteins. The last possibility was that diffuse leakage on FA corresponds to severe disruption of the BRB that results in a higher turnover of the content of the cystoid spaces.

Pathohistologic publications raised a question about whether the cystoid spaces in vivo correspond to intracytoplasmic swelling of the Müller cells or accumulated extracellular fluids in macular oedema. ${ }^{13-15}$ ICG hyperfluorescence was delineated in the nerve fibres, suggesting at least intake of ICG into the ganglion cells. ${ }^{40}$ Based on the shape, area, and array, the welldemarcated ICG hyperfluorescence implicated ICG intake into the Müller cells. However, this hypothesis does not explain that this was limited to the macular area. In addition, higher rates of washing out of ICG-binding lipoproteins in the areas with diffuse leakage seen on FA supported the accumulation of extracellular fluids rather than intracytoplasmic swelling. Future investigates should determine whether the cystoid spaces are intracellular or extracellular.

We observed larger or smaller dark spots in the ICG hyperfluorescence. If we consider that ICG hyperfluorescence represents accumulated extracellular fluids, the dark spots might correspond to microaneurysms or fibrin in the cystoid spaces. In fact, several dark spots in the ICG hyperfluorescence were colocalized to microaneurysms seen on FA images. We reported that microaneurysms sometimes protrude into the cystoid spaces and that the contents showed heterogeneous OCT reflectivity, ${ }^{41}$ suggesting fibrin deposition in the cystoid spaces. These findings might explain the larger dark spots but not the smaller ones. If ICG hyperfluorescence means ICG intake in the Müller cells, intracellular organelles including the nuclei would correspond to the dark spots.

A few months after vitrectomy, stardust-like dot fluorescence emerged and migrated, and some reached the retinal vasculature or the margin of the optic disc, as reported in eyes with a macular hole, ${ }^{31}$ whereas well-demarcated ICG hyperfluorescence resolved. We hypothesized that this hyperfluorescence corresponded to the macrophages or microglia that phagocytosed ICGbinding lipoproteins, ICG-binding serum proteins, or the debris of apoptotic Müller cells that absorbed ICG. An experimental study in rodents found that microglia had an ICG signal after intravitreal ICG administration. ${ }^{42}$ We also often observed hyperreflective foci in the cystoid spaces with higher reflectivity that might be lipid-laden macrophages and scavenge contents of the cystoid spaces. ${ }^{21,43}$ This hypothesis also explains the migration and the attachment to retinal vasculature of ICG dot fluorescence in this study. ${ }^{44}$ However, we could not exclude the possibility that the dot fluorescence was ICG intake of retinal pigment epithelial or other cells or ICG deposition in the retinal parenchyma. ${ }^{29,30}$

This retrospective study with a small number of cases had several limitations. ICG fluorescence might represent the nature of cystoid spaces in DMO after vitrectomy to some extent. However, because sub-Tenon's administration of triamcinolone was combined postoperatively, we could not determine whether vitrectomy or triamcinolone would improve the pathogenesis of DMO regarding vascular permeability and retinal thickening and concomitantly have effects on ICG fluorescence. Then, we might have to plan the clinical study in eyes treated with vitrectomy alone in order to show the effects of individual treatments.

In conclusion, we showed for the first time that the characteristics of well-demarcated ICG hyperfluorescence were related to optical coherence tomographic findings in cystoid spaces after ICG-assisted vitrectomy for DMO. 


\section{Summary}

\section{What was known before}

- ICG is observed in eyes with macular hole treated with ICG-assisted vitrectomy.

- Fluorescein pooling is associated with cystoid spaces on OCT images in diabetic macular oedema.

\section{What this study adds}

- Round- or oval-shaped ICG hyperfluorescence was delineated in eyes with diabetic macular oedema treated with ICG-assisted vitrectomy.

- ICG hyperfluorescence was mainly located in the area with hypofluorescence in FA images.

- ICG hyperfluorescence corresponded to cystoid spaces on OCT images that had higher reflectivity and hyperreflective foci.

\section{Conflict of interest}

The authors declare no conflict of interest.

\section{References}

1 Klein R, Klein BE, Moss SE, Cruickshanks KJ. The Wisconsin Epidemiologic Study of Diabetic Retinopathy. XV. The long-term incidence of macular edema. Ophthalmology 1995; 102: 7-16.

2 Gardner TW, Antonetti DA, Barber AJ, LaNoue KF, Levinson SW. Diabetic retinopathy: more than meets the eye. Surv Ophthalmol 2002; 47(Suppl 2): S253-S262.

3 Antonetti DA, Klein R, Gardner TW. Diabetic retinopathy. N Engl J Med 2012; 366: 1227-1239.

4 Mohamed Q, Gillies MC, Wong TY. Management of diabetic retinopathy: a systematic review. JAMA 2007; 298: 902-916.

5 Photocoagulation for diabetic macular edema. Early Treatment Diabetic Retinopathy Study report number 1. Early Treatment Diabetic Retinopathy Study research group. Arch Ophthalmol 1985; 103: 1796-1806.

6 Lewis H, Abrams GW, Blumenkranz MS, Campo RV. Vitrectomy for diabetic macular traction and edema associated with posterior hyaloidal traction. Ophthalmology 1992; 99: 753-759.

7 Jonas JB, Sofker A. Intraocular injection of crystalline cortisone as adjunctive treatment of diabetic macular edema. Am J Ophthalmol 2001; 132: 425-427.

8 Cunningham Jr ET, Adamis AP, Altaweel M, Aiello LP, Bressler NM, D'amico DJ et al. A phase II randomized double-masked trial of pegaptanib, an anti-vascular endothelial growth factor aptamer, for diabetic macular edema. Ophthalmology 2005; 112: 1747-1757.

9 Cogan DG, Toussaint D, Kuwabara T. Retinal vascular patterns. IV. Diabetic retinopathy. Arch Ophthalmol 1961; 66: 366-378.

10 Barber AJ, Antonetti DA, Gardner TW. Altered expression of retinal occludin and glial fibrillary acidic protein in experimental diabetes. The Penn State Retina Research Group. Invest Ophthalmol Vis Sci 2000; 41: 3561-3568.

11 Murakami T, Frey T, Lin C, Antonetti DA. Protein kinase cbeta phosphorylates occludin regulating tight junction trafficking in vascular endothelial growth factor-induced permeability in vivo. Diabetes 2012; 61: 1573-1583.

12 Joussen AM, Smyth N, Niessen C. Pathophysiology of diabetic macular edema. Dev Ophthalmol 2007; 39: 1-12.

13 Tso MO. Pathology of cystoid macular edema. Ophthalmology 1982; 89: 902-915.

14 Wolter JR. The histopathology of cystoid macular edema. Albrecht Von Graefes Arch Klin Exp Ophthalmol 1981; 216: 85-101.

15 Yanoff M, Fine BS, Brucker AJ, Eagle RC Jr. Pathology of human cystoid macular edema. Surv Ophthalmol 1984; 28(Suppl): 505-511.

16 Otani T, Kishi S. Correlation between optical coherence tomography and fluorescein angiography findings in diabetic macular edema. Ophthalmology 2007; 114: 104-107.

17 Bolz M, Ritter M, Schneider M, Simader C, Scholda C, Schmidt-Erfurth U. A systematic correlation of angiography and high-resolution optical coherence tomography in diabetic macular edema. Ophthalmology 2009; 116: 66-72.

18 Murakami T, Nishijima K, Akagi T, Uji A, Horii T, Ueda-Arakawa $\mathrm{N}$ et al. Optical coherence tomographic reflectivity of photoreceptors beneath cystoid spaces in diabetic macular edema. Invest Ophthalmol Vis Sci 2012; 53: 1506-1511.

19 Browning DJ, Altaweel MM, Bressler NM, Bressler SB, Scott IU. Diabetic macular edema: what is focal and what is diffuse? Am J Ophthalmol 2008; 146: 649-655

20 Murakami T, Nishijima K, Sakamoto A, Ota M, Horii T, Yoshimura N. Foveal cystoid spaces are associated with enlarged foveal avascular zone and microaneurysms in diabetic macular edema. Ophthalmology 2011; 118: 359-367.

21 Horii T, Murakami T, Nishijima K, Akagi T, Uji A, Arakawa N et al. Relationship between fluorescein pooling and optical coherence tomographic reflectivity of cystoid spaces in diabetic macular edema. Ophthalmology 2012; 119: 1047-1055.

22 Kadonosono K, Itoh N, Uchio E, Nakamura S, Ohno S. Staining of internal limiting membrane in macular hole surgery. Arch Ophthalmol 2000; 118: 1116-1118.

23 Gandorfer A, Messmer EM, Ulbig MW, Kampik A. Resolution of diabetic macular edema after surgical removal of the posterior hyaloid and the inner limiting membrane. Retina 2000; 20: 126-133.

24 Haller JA, Qin H, Apte RS, Beck RR, Bressler NM, Browning DJ et al. Vitrectomy outcomes in eyes with diabetic macular edema and vitreomacular traction. Ophthalmology 2010; 117: 1087-1093; e3.

25 Yamamoto T, Hitani K, Sato Y, Yamashita H, Takeuchi S. Vitrectomy for diabetic macular edema with and without internal limiting membrane removal. Ophthalmologica 2005; 219: 206-213

26 Patel JI, Hykin PG, Schadt M, Luong V, Fitzke F, Gregor ZJ. Pars plana vitrectomy with and without peeling of the inner limiting membrane for diabetic macular edema. Retina 2006; 26: 5-13.

27 Almony A, Nudleman E, Shah GK, Blinder KJ, Eliott DB, Mittra RA et al. Techniques, rationale, and outcomes of internal limiting membrane peeling. Retina 2012; 32: 877-891.

28 Gass CA, Haritoglou C, Schaumberger M, Kampik A. Functional outcome of macular hole surgery with and without indocyanine green-assisted peeling of the internal limiting membrane. Graefes Arch Clin Exp Ophthalmol 2003; 241: 716-720. 
29 Ciardella AP, Schiff W, Barile G, Vidne O, Sparrow J, Langton $\mathrm{K}$ et al. Persistent indocyanine green fluorescence after vitrectomy for macular hole. Am J Ophthalmol 2003; 136: 174-177.

30 Weinberger AW, Kirchhof B, Mazinani BE, Schrage NF. Persistent indocyanine green (ICG) fluorescence 6 weeks after intraocular ICG administration for macular hole surgery. Graefes Arch Clin Exp Ophthalmol 2001; 239: 388-390.

31 Sayanagi K, Ikuno Y, Soga K, Sawa M, Oshima Y, Kamei M et al. Residual indocyanine green fluorescence pattern after vitrectomy for idiopathic macular hole with internal limiting membrane peeling. Br J Ophthalmol 2007; 91: 939-944.

32 Tadayoni R, Paques M, Girmens JF, Massin P, Gaudric A. Persistence of fundus fluorescence after use of indocyanine green for macular surgery. Ophthalmology 2003; 110: 604-608.

33 Ashikari M, Ozeki H, Tomida K, Sakurai E, Tamai K, Ogura Y. Retention of dye after indocyanine green-assisted internal limiting membrane peeling. Am J Ophthalmol 2003; 136: 172-174.

34 Sekiryu T, lida T. Long-term observation of fundus infrared fluorescence after indocyanine green-assisted vitrectomy. Retina 2007; 27: 190-197.

35 Aiello LP, Beck RW, Bressler NM, Browning DJ, Chalam KV, Davis $\mathrm{M}$ et al. Rationale for the diabetic retinopathy clinical research network treatment protocol for center-involved diabetic macular edema. Ophthalmology 2011; 118: e5-e14.

36 Yoneya S, Saito T, Komatsu Y, Koyama I, Takahashi K, Duvoll-Young J. Binding properties of indocyanine green in human blood. Invest Ophthalmol Vis Sci 1998; 39: 1286-1290.
37 Baker KJ. Binding of sulfobromophthalein (BSP) sodium and indocyanine green (ICG) by plasma alpha-1 lipoproteins. Proc Soc Exp Biol Med 1966; 122: 957-963.

38 Cherrick GR, Stein SW, Leevy CM, Davidson CS. Indocyanine green: observations on its physical properties, plasma decay, and hepatic extraction. J Clin Invest 1960; 39: 592-600.

39 Horiguchi M, Nagata S, Yamamoto N, Kojima Y, Shimada Y. Kinetics of indocyanine green dye after intraocular surgeries using indocyanine green staining. Arch Ophthalmol 2003; 121: 327-331.

40 Iriyama A, Uchida S, Yanagi Y, Tamaki Y, Inoue Y, Matsuura K et al. Effects of indocyanine green on retinal ganglion cells. Invest Ophthalmol Vis Sci 2004; 45: 943-947.

41 Horii T, Murakami T, Nishijima K, Sakamoto A, Ota M, Yoshimura N. Optical coherence tomographic characteristics of microaneurysms in diabetic retinopathy. Am J Ophthalmol 2010; 150: 840-848.

42 Paques M, Simonutti M, Augustin S, Goupille O, El Mathari B, Sahel JA. In vivo observation of the locomotion of microglial cells in the retina. Glia 2010; 58: 1663-1668.

43 Bolz M, Schmidt-Erfurth U, Deak G, Mylonas G, Kriechbaum K, Scholda C. Optical coherence tomographic hyperreflective foci: a morphologic sign of lipid extravasation in diabetic macular edema. Ophthalmology 2009; 116: 914-920.

44 Chen L, Yang P, Kijlstra A. Distribution, markers, and functions of retinal microglia. Ocul Immunol Inflamm 2002; 10: $27-39$.

Supplementary Information accompanies this paper on Eye website (http://www.nature.com/eye) 